\title{
Dieta natural do siri-azul Callinectes sapidus (Decapoda, Portunidae) na região estuarina da Lagoa dos Patos, Rio Grande, Rio Grande do Sul, Brasil
}

\author{
Alexandre Oliveira, Taciana K. Pinto, Débora P. D. Santos \& Fernando D’Incao
}

Fundação Universidade Federal do Rio Grande, Caixa Postal 474, 96201-900 Rio Grande, RS, Brasil. (alexandre@octopus.furg.br, taciana@dern.ufes.br,debora@hotmail.com,docdinca@furg.br)

\begin{abstract}
Natural diet of the blue crab Callinectes sapidus (Decapoda, Portunidae) in the Patos Lagoon estuary area, Rio Grande, Rio Grande do Sul, Brazil. The Southern Brazil blue crab Callinectes sapidus Rathbun, 1869 is the most abundant crab of the genus Callinectes in Patos Lagoon estuary. Although this species is widely distributed throughout the Patos Lagoon estuary area, there is little information about its natural diet. This species is an important predator and has a significant influence on its prey populations. The aim of this study was to check the natural diet of blue crab through the foregut contents analysis. Crabs were collected using an otter trawl net from March 2003 to March 2004. After collected, crabs were preserved immediately in $10 \%$ formaldehyde. The carapace width, weight and sex were measured for each individual. The foregut of each crab was removed and stored in $70 \%$ ethanol. Blue crab feeds on a wide variety of sessile and slow moving invertebrates. The main item was Detritus, followed by the suspension-feeder mollusk Erodona mactroides Bosc, 1802 (Erodonidae). Sand grains and the small crustaceans of class Ostracoda, were an important component of the foregut contents, but sand grains were not considered food. Setae and pieces of Polychaeta and some seeds of submersed higher vegetation of Ruppia maritima L. (Potamogatonaceae) (beaked tasselweed), and Zannichellia palustris L. (Potamogatonaceae) (homed pondweed), were also found. This study has as purpose to provide information for a long-term protection and conservation of blue crab population and also to characterize its relationship with the macrobenthic communities in the Patos Lagoon estuary.
\end{abstract}

KEYWORDS. Feeding ecology, Portunidae, Callinectes, blue crab, Patos Lagoon estuary.

RESUMO. Na região estuarina da Lagoa dos Patos, o siri-azul Callinectes sapidus Rathbun, 1869 é o mais abundante dentre as espécies do gênero. Apesar do siri-azul exercer influência sobre as comunidades bentônicas, por ser considerado um predador do topo da cadeia alimentar, não existem muitos estudos sobre aspectos ecológicos, inclusive sobre a composição e variabilidade sazonal de sua dieta natural, neste estuário. O objetivo principal deste estudo foi investigar a composição da dieta do siri-azul, evidenciando possíveis relações com a comunidade bentônica da região estuarina da Lagoa dos Patos. O período de estudo foi de março de 2003 a março de 2004, com coletas bimestrais. Os organismos foram coletados com auxílio de rede de arrasto de fundo, com malha de $13 \mathrm{~mm}$ entre nós opostos. Ainda em campo os animais foram fixados em formol 10\%. Após a coleta os animais foram separados quanto ao sexo, medidos (largura e comprimento da carapaça $-\mathrm{cm}$ ) e pesados (peso $-\mathrm{g}$ ). Após a triagem, os animais foram dissecados e os intestinos retirados e pesados. As análises dos conteúdos alimentares dos siris demonstraram que os hábitos alimentares são diversificados, constituindo-se principalmente de invertebrados bentônicos. O item encontrado com maior freqüência foi Detrito, seguido pelo molusco filtrador Erodona mactroides Bosc, 1802 (Erodonidae). Crustáceos da classe Ostracoda e grãos de areia foram importantes componentes dos conteúdos dos intestinos anteriores, sendo que areia não foi considerada como item alimentar, propriamente dito. Também foram encontradas cerdas e mandíbulas de poliquetos, além de sementes das macrófitas Ruppia maritima L. (Potamogatonaceae) e Zannichellia palustris L. (Potamogatonaceae). Este estudo serve como subsídio para medidas de proteção e conservação da população do siri-azul, bem como caracteriza relações tróficas com comunidades bentônicas do estuário da Lagoa dos Patos.

PALAVRAS-CHAVE. Ecologia alimentar, Portunidae, Callinectes, siri-azul, estuário da Lagoa dos Patos.

O siri-azul Callinectes sapidus Rathbun, 1896 ocorre na costa leste do Oceano Atlântico, em estuários, lagunas, deltas de rios e regiões oceânicas adjacentes desde Massachusetts (EUA) até o Uruguai. Ocasionalmente sua distribuição é ampliada, alcançando Nova Scotia (Canadá) e Argentina (Williams, 1974; Boschi, 1982; Melo, 1996). O siri-azul possui padrão de distribuição disjunto, ou seja, apresenta uma área de distribuição norte e outra sul, sendo separada por um hiato de pelo menos $50^{\circ}$ de latitude, que ocorre no Golfo do México (Melo, 1996). A espécie é considerada ainda como o braquiúro mais importante, do ponto de vista pesqueiro, da região estuarina da Lagoa dos Patos, RS (CEPERG/IBAMA, 2002).

Por serem vorazes predadores, os braquiúros podem exercer importantes efeitos sobre a estrutura e função de sistemas aquáticos e afetar diretamente a abundância e a estrutura de tamanhos de suas presas, através da remoção selecionada de certos indivíduos da comunidade predada (SIH et al., 1985; Hines et al., 1990; Wright et al., 1996). O conhecimento de aspectos da biologia da alimentação dos braquiúros é de fundamental importância, pois a disponibilidade e utilização do alimento desempenham importantes papéis em seus padrões de distribuição, migração, ecdise e reprodução (McLAUGHLIN \& Hebard, 1961; Laughlin, 1982; Hines \& Ruiz, 1995).

Devido a seus hábitos alimentares, $C$. sapidus constitui-se num predador bentônico chave por controlar a abundância de outras espécies bentônicas estuarinas, consumindo uma grande variedade de organismos tanto da infauna como epifauna, tais como bivalves, poliquetos, crustáceos, entre outros (TAGATZ, 1968; BLUNDON \& KenNedy, 1982; Fitz \& WiegerT, 1990; Hines et al., 1990). LAUGHLin (1982), em estudos sobre a dieta de C. sapidus em Apalachicola Bay, Flórida, determinou que a dieta deste siri varia conforme seu hábitat, estação do ano, 
tamanho e distribuição dos organismos dos quais se alimenta. Porém, segundo este mesmo autor, aspectos que levam em consideração a partilha, utilização dos recursos e a potencial relação com as distribuições temporais e espaciais das espécies, têm sido deixados à parte.

Kapusta \& Bemvenuti (1998) verificaram que juvenis do siri-azul apresentavam comportamento alimentar generalista-oportunista em uma pradaria da macrófita aquática Ruppia maritima L. e num plano nãovegetado de uma enseada estuarina da Lagoa dos Patos, RS. No entanto, estes resultados podem estar subestimados, visto que os exemplares de C. sapidus foram, principalmente, juvenis.

Apesar da dieta do siri-azul ter sido objeto de vários estudos (entre outros, Laughlin, 1982; Fitz \& Wiegert, 1990; Hsueh et al., 1992; Kapusta \& Bemvenuti, 1998) nas diversas áreas em que está presente, existe, principalmente no Brasil, falta de informação sobre a influência de fatores ambientais (como a ocorrência sazonal dos itens alimentares) na dieta natural desta espécie. O presente trabalho pretende contribuir no preenchimento destas lacunas, estudando variações sazonais da dieta natural de C. sapidus através da análise de seus hábitos alimentares, investigando as relações tróficas da espécie na região estuarina da Lagoa dos Patos, RS.

\section{MATERIAL E MÉTODOS}

As coletas foram bimestrais durante o período de março de 2003 a março de 2004 (exceto no mês de janeiro de 2004, devido a problemas operacionais na embarcação), em uma área estuarina da Lagoa dos Patos (32 $\left.2^{\circ} 00^{\prime}-32^{\circ} 03^{\prime} \mathrm{S} ; 052^{\circ} 11^{\prime}-052^{\circ} 13^{\prime} \mathrm{W}\right)$. O método de captura foi o arrasto de portas (malhas da região do saco medindo $13 \mathrm{~mm}$ e sobre-saco $5 \mathrm{~mm}$, entre nós opostos), com duração de cinco minutos e velocidade média de 1,6 nós. Imediatamente após a coleta, os animais foram fixados em formalina a $10 \%$ e conduzidos ao Laboratório de Crustáceos Decápodos da FURG para a biometria e análise dos conteúdos alimentares.

Por considerarmos a terminologia sugerida por McLaughlin (1983), intestino anterior, para a denominação da estrutura que possui e armazena o alimento logo após a ingestão pelo siri-azul, o termo mais correto e fidedigno às atribuições e funções desempenhadas por tal estrutura, este será utilizado ao longo deste estudo.

Em laboratório, os animais foram separados por sexo e seu grau de desenvolvimento (juvenil ou adulto) verificado, o qual obtido pela observação do formato e constatação da aderência do abdômen aos esternitos torácicos (TAISSOUN, 1969).

A biometria dos animais constou da medida da largura e comprimento da carapaça (em cm), com auxílio de paquímetro digital $(0,01 \mathrm{~mm})$. A largura da carapaça (LC) foi considerada como a distância entre os últimos dentes ânterolaterais e o comprimento da carapaça (CC) como a distância entre o espinho epistomial e a margem posterior da carapaça. Cada animal foi pesado em balança de precisão (em gramas). Foram utilizados apenas indivíduos em intermuda, sendo rejeitados os indivíduos em ecdise, como sugerido por HAEFNER (1990).

Após a biometria, o intestino anterior foi retirado através de uma sutura na forma de U na região gástrica, e mantido em álcool a $70 \%$ até o momento da análise. Cada intestino foi pesado e avaliado visualmente em relação ao grau de repleção (quantidade de alimento presente no seu interior) e classificado com base em HAEFNER (1990), modificado por KAPUSTA \& BeMVENUTI (1998), sendo designados às seguintes classes: classe 3 , cheio $(\leqslant 100 \%$ e $>70 \%$ ); classe 2 , semicheio ( $\leqslant 70 \%$ e $>30 \%)$; classe 1 , semivazio $(\leqslant 30 \%$ e $>1 \%)$; classe 0 , vazio $(\leqslant 1 \%)$. Após a classificação, os intestinos foram abertos e seu conteúdo depositado em placa de Petri. Os intestinos foram secos em papel absorvente e novamente pesados. A classificação anterior foi conferida e corrigida, se necessário, após a abertura dos intestinos. O conteúdo alimentar foi identificado sob microscópio estereoscópio até o menor nível taxonômico possível. O material identificado se encontra armazenado no Laboratório de Crustáceos Decápodes, Departamento de Oceanografia, Fundação Universidade Federal do Rio Grande, Rio Grande do Sul.

Após a identificação e classificação, alguns itens alimentares foram agrupados para uma melhor análise e compreensão dos resultados obtidos. Assim, ostrácodes, anfípodes, isópodos e cirripédios formaram o grupo denominado Outros Crustáceos.

O método dos Pontos, proposto por Williams (1981), avalia a contribuição relativa de cada item, no volume total do conteúdo do intestino, sendo subjetivamente atribuída uma escala de pontos com cinco categorias. O item recebeu 100 pontos apresentando valores de volume entre $100 \%$ e $\geqslant 95 \%$ do conteúdo do intestino; 75 pontos entre $<95 \%$ e $\geqslant 65 \%$; 50 pontos entre $<65$ e $\geqslant 35 \% ; 25$ pontos entre $<35$ e $\geqslant 5 \%$; e 2,5 pontos para volumes com valores $<5 \%$. O número de pontos recebidos por cada item foi relativo ao grau de repleção do intestino no qual se encontrava. Esta atribuição consiste em multiplicar o número de pontos por um valor dependente das classes de repleção: classe 3, 1,00; classe 2, 0,60; classe 1,0,20. Assim, o máximo de pontos que um simples item em um único intestino pode obter é 100 ( 100 x 1,00, onde está presente somente um item em um intestino completamente cheio). O mínimo possível é 0,5 ( 2,5 x 0,20, onde o item representa menos de $5 \%$ de um intestino semivazio). Os intestinos da classe 0 foram desconsiderados por não possuírem nenhum item alimentar em seu interior.

A porcentagem para cada item foi calculada segundo a fórmula abaixo:

Porcentagem de Pontos (P\%) para cada $i$ item

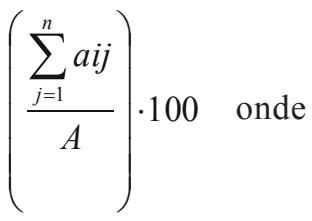

$a_{i j}$ é o número de pontos que cada item $i$ obteve no intestino $j$ de cada animal e $A$ o número total de pontos para todos os itens em todos os animais da amostra.

A freqüência de ocorrência corresponde à freqüência percentual do número de intestinos onde 
ocorre determinado item alimentar em relação ao número total de intestinos com alimento, sendo calculada segundo a expressão abaixo (WiLliams, 1981):

Porcentagem da Freqüência de Ocorrência $(\mathrm{O} \%)=$

$$
\left(\frac{b_{i}}{N}\right) \cdot 100 \text { onde }
$$

$b_{i}$ é o número de animais nos quais o item $i$ está presente e $N$, o número de animais da amostra (todos os que possuem conteúdo no intestino).

A correlação de Spearman (ZAR, 1984) foi utilizada para a comparação entre os resultados das porcentagens dos Pontos ( $\mathrm{P} \%)$ e Freqüência de Ocorrência $(\mathrm{O} \%)$ dos itens alimentares ao longo das estações do ano. As freqüências relativas dos itens alimentares consumidos por sexo, bem como as porcentagens dos intestinos anteriores em cada classe de repleção, foram comparadas utilizando-se o teste do $\chi^{2}(\mathrm{p}<0,05)$ (Ludwig \& ReYNOLDS, 1988).

\section{RESULTADOS E DISCUSSÃO}

Foram analisados 997 siris, sendo 542 machos $(54,36 \%)$ e 455 fêmeas $(45,64 \%)$. A análise do $\chi^{2}(p<0,05)$ aplicada à proporção dos sexos não demonstrou diferença significativa $\left(\chi^{2}=0,76 \mathrm{df}=1 ; p=0,38\right)$. A largura média da carapaça dos siris foi de $5,63 \pm 0,09 \mathrm{~cm}$ para machos e $5,56 \pm 0,09 \mathrm{~cm}$ para fêmeas, enquanto que o comprimento médio da carapaça foi de $3,15 \pm 0,05 \mathrm{~cm}$ para machos e $3,08 \pm 0,09 \mathrm{~cm}$ para fêmeas. Já o peso dos animais foi de $25,4 \pm 1,22 \mathrm{~g}$ para machos e 22,1 $\pm 1,05 \mathrm{~g}$ para fêmeas. Aproximadamente 90\% (n = 894) dos intestinos de $C$. sapidus apresentaram algum tipo de conteúdo alimentar, sendo este valor significativamente mais alto $\left(\chi^{2}=62,95\right.$ df $=1 ; p<0,01)$ do que o dos intestinos vazios que representaram, aproximadamente, $10 \%(\mathrm{n}=103)$. Os machos apresentaram 488 intestinos com itens alimentares $(90,04 \%)$ e 54 vazios $(9,96 \%)$. Para as fêmeas, o número de intestinos que apresentaram algum tipo de alimento foi de $406(89,23 \%)$ enquanto que o número de vazios foi de $49(10,77 \%)$. A análise do $\chi^{2}(p<0,05)$ aplicada aos intestinos que apresentaram algum tipo de item alimentar, em relação à proporção dos sexos, também demonstrou diferenças significativas tanto para machos $\left(\chi^{2}=67,13 \mathrm{df}=1 ; p<0,01\right)$, quanto para fêmeas $\left(\chi^{2}=61,56\right.$ $\mathrm{df}=1 ; \mathrm{p}<0,01)$. Laughlin (1982), no estuário de Apalachicola, Flórida (EUA) e Hsuen et al. (1992), em fundos lamosos de Mobile Bay, Alabama (EUA), registraram proporções similares de intestinos. Valores semelhantes também foram observados para outras espécies do gênero, como $C$. danae na Lagoa da Conceição, Santa Catarina (Branco \& Verani, 1997) e $C$. ornatus na Baía de Ubatuba, São Paulo (MANTELATto \& Christofoletti, 2001) e na Baía de Itapocoroy (Branco et al., 2002).

A alta porcentagem de intestinos anteriores de $C$. sapidus com alimento indica uma alta taxa de predação/ alimentação desta espécie. De acordo com EdGAR (1990), os portunídeos apresentam maior atividade e crescimento que a maioria dos crustáceos, podendo ser esta a explicação para a constante voracidade relatada em estudos alimentares com espécies desta família.

A análise dos conteúdos alimentares revelou uma ampla variedade de itens componentes da dieta do siriazul, o qual apresentou predação sobre seis táxons, dentre eles Pisces e Plantae. A maior diversidade alimentar foi observada para o grupo Crustacea, seguidas por Mollusca e Plantae (Tab. I).

Características similares da alimentação também foram observadas em outras espécies do gênero, como predadoras oportunistas (TAGATZ, 1968; PAUL, 1981;

Tabela I. Itens alimentares na dieta natural do siri-azul Callinectes sapidus durante o período de março de 2003 a março de 2004 , na região estuarina da Lagoa dos Patos, Rio Grande, RS. *Tipos de peças baseados em Williams (1981) e Haefner (1990).

\begin{tabular}{|c|c|c|c|}
\hline \multicolumn{2}{|c|}{ Item Alimentar } & \multirow{2}{*}{$\begin{array}{l}\text { *Tipos de peças encontradas nos } \\
\text { intestinos anteriores }\end{array}$} & \multirow[t]{2}{*}{ Espécie } \\
\hline Plantae & & & \\
\hline & Zannichelliaceae & Semente, restos & Zannichellia palustris L. \\
\hline & Ruppiaceae & Semente, restos & Ruppia maritima $\mathrm{L}$. \\
\hline \multicolumn{4}{|c|}{ Mollusca } \\
\hline & Bivalvia & Partes das conchas, bisso & Erodona mactroides Bosc, 1802 \\
\hline & Gastropoda & Animais inteiros, conchas vazias & Heleobia australis (Marcus \& Marcus, 1963) \\
\hline \multicolumn{4}{|c|}{ Crustacea } \\
\hline & Ostracoda & Animais inteiros & Ostrácode indet. \\
\hline & Amphipoda & Animais inteiros & Anfípode indet. \\
\hline & Isopoda & Animais inteiros & Uromunna peterseni Pires, 1985 \\
\hline & Balanomorpha & Partes da carapaça & Balanus sp. \\
\hline & Tanaidacea & Animais inteiros & Tanais stanfordi (Richardson, 1901) \\
\hline & & & Kalliapseudes schubartii Mañe-Garzón, 1979 \\
\hline & Dendrobranchiata & Partes da carapaça e télson & Farfantepenaeus paulensis Peréz-Farfante, 1967 \\
\hline & Pleocyemata & Partes da carapaça e quelas & Callinectes sp. \\
\hline & & & Palaemonetes argentinus Nobili, 1901 \\
\hline \multicolumn{4}{|c|}{ Annelida } \\
\hline & Polychaeta & Mandíbulas, cerdas e partes corporais & \\
\hline Insecta & & Asas & \\
\hline \multicolumn{4}{|l|}{ Pisces } \\
\hline & Teleostei & Escamas, ossos, otólitos & \\
\hline Areia & & Grãos finos e grossos & \\
\hline Detrito & & Material de origem animal e vegetal & \\
\hline
\end{tabular}


Hsueh et al., 1992; Branco et al., 2002), detritívoras (Carqueija \& Gouvêa, 1998), ou como onívoras (LAughlin, 1982; Rosas et al., 1994; Chazaro-Olvera et al., 2000; Mantelatto \& Christofoletti, 2001). Kapusta \& BEMVENUTI (1998) atribuem ainda um comportamento alimentar generalista-oportunista para juvenis de $C$. sapidus. Outros portunídeos apresentaram dietas semelhantes à de C. sapidus, como Scylla serrata (Forskal, 1775) (Hill, 1976), Portunus pelagicus (Linnaeus, 1766) (Williams, 1982), Ovalipes catharus (White, 1843) (WeAR \& Haddon, 1987), Necora puber (Linnaeus, 1767) (FreIrE \& GonZÁLEZ-GURRIARÁN, 1995) e Liocarcinus depurator (Linnaeus, 1758) (FREIRE, 1996).

Este comportamento reflete a composição bentônica da área em que foram realizados, ou seja, o que está mais disponível e acessível ao predador (WiLliams, 1982). KAPUSTA \& BEMVENUTI (1998), estudando a atividade nictemeral da alimentação de juvenis de $C$. sapidus numa pradaria e num plano não-vegetado, observaram a dominância de itens alimentares de acordo com os distintos hábitats.

Visto que os braquiúros (Fitz \& WIEGERT, 1991) e mais precisamente, os portunídeos (STONER \& BUCHANAN, 1990; HsueH et al., 1992) são considerados controladores da abundância e diversidade de bivalves, bem como da comunidade bentônica em geral, é de se esperar que sua dieta esteja diretamente ligada à composição da fauna dos fundos das regiões que habitam. Os crustáceos decápodes foram considerados, em conjunto com os peixes, predadores responsáveis por manter a macrofauna bentônica de fundos inconsolidados abaixo da capacidade de suporte do sistema estuarino da Lagoa dos Patos (Bemvenuti, 1987). Experimentos em laboratório têm confirmado que $C$. sapidus regula a população de bivalves e peixes, através da capacidade de selecionar tamanhos específicos de presas (SEED, 1980; BLUNDON \& KenNedy, 1982; West \& Williams, 1986).

Houve diferença significativa quanto ao grau de repleção dentro de cada mês de coleta, em todos os meses coletados $(\mathrm{p}<0,05)$. As maiores porcentagens do grau de repleção cheio, em março e maio de 2003, podem estar relacionadas diretamente com um período de maior atividade do siri, coincidindo com temperaturas mais elevadas nestes meses $\left(26,80 \pm 0,87^{\circ} \mathrm{C}\right.$ e $18,82 \pm 2,54^{\circ} \mathrm{C}$, respectivamente). Já em julho e setembro, onde temperaturas mais baixas foram predominantes $(15,60 \pm$ $0,46^{\circ} \mathrm{C}$ e $15,97 \pm 1,76^{\circ} \mathrm{C}$, respectivamente), não foram observadas porcentagens dominantes entre os graus de repleção (Fig. 1). Nesta análise não foi considerado o grau de repleção vazio. Resultados similares foram observados por MANTELATTO \& CHRISTOFOLETTI (2001) para C. ornatus.

No período de inverno, o siri-azul apresenta mobilidade reduzida e conseqüentemente se alimenta com pouca freqüência, ficando a maior parte do tempo enterrado no sedimento (Schaffner \& Diaz, 1988). Por outro lado, em novembro de 2003 e março de 2004, as maiores porcentagens foram observadas no grau de repleção semivazio, apesar das temperaturas elevadas $\left(24,50 \pm 0,58^{\circ} \mathrm{C}\right.$ e $23,45 \pm 0,54^{\circ} \mathrm{C}$, respectivamente).

As freqüências totais de ocorrência dos itens alimentares de C. sapidus (Tab. II) indicam que este siri se alimenta principalmente dos grupos Mollusca (Erodona mactroides Bosc, 1802 e Heleobia australis (Marcus \& Marcus 1963)) e Crustacea (ostrácodes, anfípodes, isópodos, cirripédios, tanaidáceos e decápodes). O item Detrito, em conjunto com os dois anteriores, são os itens de maior freqüência de ocorrência

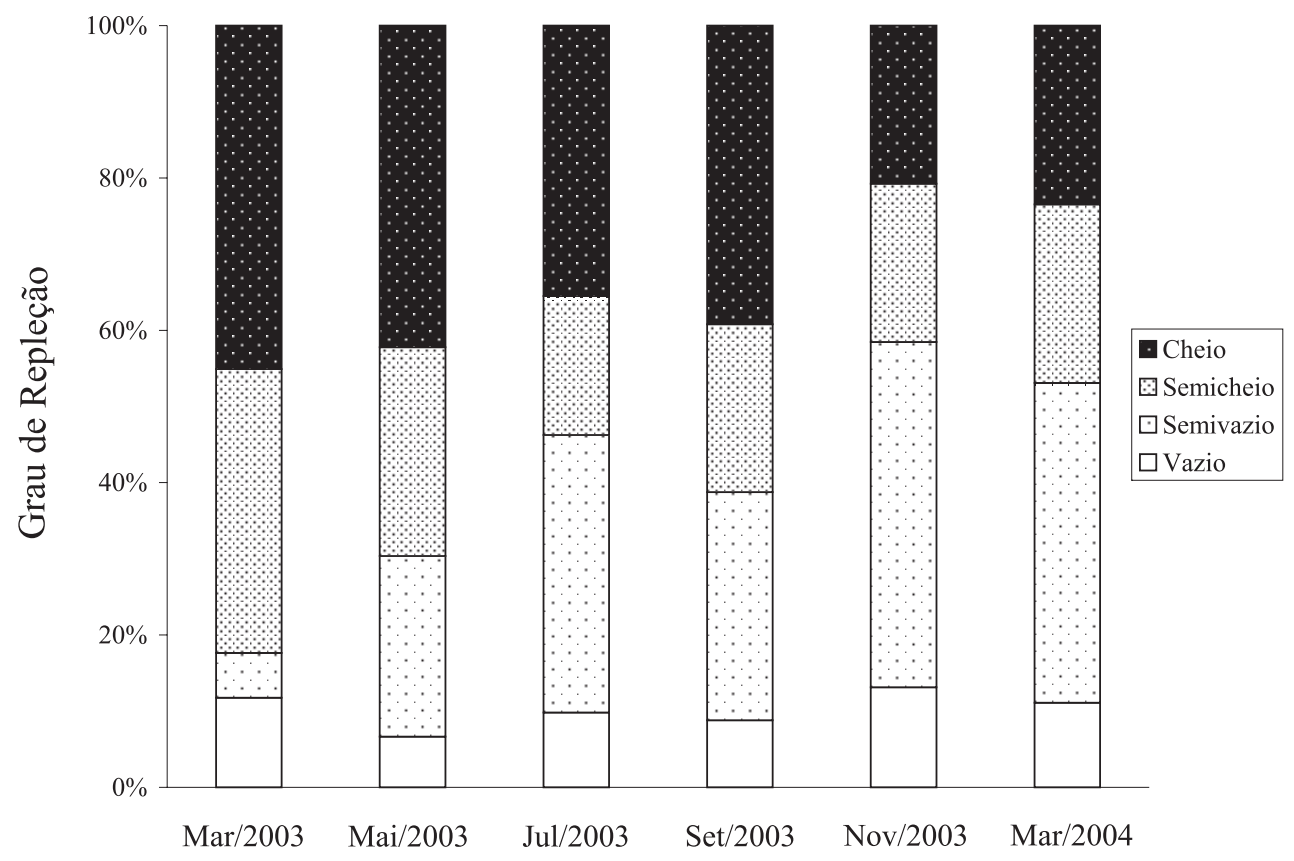

Fig. 1. Grau de repleção dos intestinos anteriores de Callinectes sapidus, durante o período de março de 2003 a março de 2004 , na região estuarina da Lagoa dos Patos, RS. 
nos intestinos anteriores de C. sapidus (Tab. II). Devido à alta correlação entre as porcentagens dos Pontos e Freqüência de Ocorrência $(r=0,90)$, as análises serão baseadas nas porcentagens dos Pontos. Somente os grupos Mollusca e Crustacea são responsáveis por mais de $57 \%$ (porcentagem acumulada) dos itens alimentares reconhecidos e identificados nos intestinos anteriores do siri-azul (Tab. II). Isto demonstra a importância destes organismos, bem como do siri-azul, na teia trófica da região estuarina da Lagoa dos Patos.

Quando a porcentagem do item Detrito é adicionada a esse número, a porcentagem acumulada sobe para aproximadamente $80 \%$ dos itens do qual o siri se alimenta. Resultados similares foram obtidos para a mesma espécie por Darnell (1958), LaUghlin (1982), Hsueh et al. (1992) e Kapusta \& Bemvenuti (1998); PAul (1981) e Ditrel (1993) para Callinectes arcuatus (Ordway, 1863); Haefner (1990) e Mantelatto \& Christofoletti (2001) para C. ornatus; e Branco \& VERANI (1997) para $C$. danae.

Apesar de Annelida e Plantae apresentarem baixos valores na porcentagem dos pontos, estes itens obtiveram porcentagens de freqüência de ocorrência acima dos 35\%, demonstrando sua importância como itens da dieta natural deste siri (Tab. II). Annelida foi o principal item observado nos conteúdos alimentares de $C$. danae em Santa Catarina, Brasil (BRANCo \& VerANI, 1997), estando entre os quatro mais importantes itens presentes na dieta natural de C. ornatus (HAEFner, 1990) e C. sapidus (KAPUSta \& Bemvenuti, 1998). Já Plantae foi citado apenas como recurso secundário, utilizado nas fases iniciais do desenvolvimento do siri-azul, não estando entre os quatro itens de maior importância da dieta natural de C. ornatus (Mantelatto \& Christofoletti, 2001).

Pisces e Insecta representaram apenas 1,3\% dos itens reconhecidos e identificados da dieta de C. sapidus, durante o período de estudo (Tab. II). Diferente destes resultados, alguns autores reportam que Pisces está entre os quatro principais itens alimentares do siri-azul (TAGATZ, 1968; Laughlin, 1982), e até mesmo como principal item de $C$. sapidus em Mobile Bay (Hsuen et al., 1992). Porém, estes mesmos autores atentam para uma provável interferência de rejeitos de pesca próximo aos locais de estudo.

Detrito foi o mais freqüente item alimentar durante o período de estudo (Tab. II). A composição deste item abrange restos digeridos de origem animal e vegetal, que ainda não foram absorvidos pelo intestino médio deste animal (DALL \& MORIARTY, 1983). Sua presença pode estar refletindo alta freqüência de alimentação e rápidos processos de desgaste e maceração das presas, que ocorrem principalmente no intestino anterior dos crustáceos (Dall \& Moriarty, 1983). Esse item também foi o mais freqüente na dieta de C. ornatus (MANTELATTO \& Christofoletti, 2001), chegando a quase $100 \%$ de freqüência de ocorrência nestes animais. CHAZARO-OLVERA et al. (2000) observaram, com o aumento no tamanho, uma diminuição de detrito e um aumento na quantidade de crustáceos e moluscos na dieta de C. similis Williams, 1966.

Isto pode estar ocorrendo também com C. sapidus, visto que a maioria dos intestinos analisados pertencia a indivíduos em estádio juvenil. Alguns autores não tratam especificamente desta categoria, ou por a considerarem em conjunto com outros itens (D'InCAO et al., 1990; CARqueiJa \& GouvÊA, 1998; Branco et al., 2002), ou por este item não obter freqüências que aparentemente possam justificar sua discussão (Rosas et al., 1994; Branco \& Verani, 1997; Chazaro-Olvera et al., 2000).

Mollusca foi o grupo mais freqüente predado pelo siri-azul, sendo que E. mactroides contribuiu com mais do que o quádruplo da freqüência de ocorrência de $H$. australis (Tab. II). Freqüências similares deste bivalve não foram observadas na dieta de juvenis de C. sapidus numa enseada estuarina da Lagoa dos Patos (KAPUSTA \& BemVENUTI, 1998), onde os autores verificaram maior freqüência do gastrópode $H$. australis. Esta freqüência, ainda segundo esses autores, está ligada às altas densidades deste gastrópode registradas neste local.

A alta freqüência de $E$. mactroides registrada neste estudo pode estar relacionada a elevadas taxas de densidade deste bivalve para a área (BEMVENUTI, 1998) onde os siris foram coletados. As freqüências indicam que há ingestão maior do bivalve em relação ao gastrópode, sugerindo uma estratégia comum aos predadores, na qual se obtém maior ganho energético através da seleção de presas maiores (REISE, 1985). Este comportamento também foi observado por WRIGHT et al. (1996) em estudos de predação seletiva do siri-azul sobre o gastrópode Bittium varium Pfeiffer, 1840. Por outro lado, HugHes \& SEED (1981) observaram que o siri-azul prefere bivalves de menor tamanho àqueles maiores de Geukensia (=Modiolus) demissa (Dillwyn, 1817). De qualquer forma, a presença de moluscos na dieta dos braquiúros, em especial portunídeos, é de grande importância e foi registrada por vários autores (PAUL, 1981; LAUGHLin, 1982; WeAR \& HADDON, 1987; Hines et al., 1990; Hsuen et al., 1992; CARqueIJA \& GouvÊA, 1998; ChazaroOlvera et al., 2000; Mantelatto \& Christofoletti, 2001; BRANCO et al., 2002).

O grupo Crustacea foi o terceiro item de maior freqüência de ocorrência na dieta alimentar de $C$. sapidus. Formado por tanaidáceos, outros crustáceos e decápodes, este grupo tem grande importância na dieta do siri-azul. A alta freqüência de ocorrência $(\mathrm{O} \%)$, de aproximadamente $80 \%$, pode ser explicada pela presença de ostrácodes, com freqüência de aproximadamente $39 \%$. Além disso, a freqüência dos tanaidáceos também contribuiu de forma acentuada para o grupo Crustacea (Tab. II); estes

Tabela II. Porcentagem total dos Pontos (P\%) e Freqüência de Ocorrência $(\mathrm{O} \%)$ dos itens alimentares presentes nos intestinos anteriores do siri-azul Callinectes sapidus na região estuarina da Lagoa dos Patos, Rio Grande, RS.

\begin{tabular}{lrr}
\hline Itens Alimentares & $\mathrm{P} \%$ & $\mathrm{O} \%$ \\
\hline Erodona mactroides & 28,2 & 71,7 \\
Detrito & 21,9 & 81,1 \\
Outros Crustáceos & 12,4 & 44,6 \\
Plantae & 8,3 & 38,4 \\
Tanaidáceos & 6,5 & 21,6 \\
Heleobia australis & 6,2 & 21,3 \\
Areia & 5,7 & 32,4 \\
Annelida & 5,6 & 35,5 \\
Decápodes & 3,9 & 14,5 \\
Pisces & 1,0 & 3,7 \\
Insecta & 0,3 & 3,8 \\
\hline
\end{tabular}


organismos estão nos fundos inconsolidados da Lagoa dos Patos, sendo considerados como espécies dominantes deste hábitat (BEMVEnUti, 1987; RosA-Filho \& Bemvenuti, 1998). Por outro lado, Kapusta \& Bemvenuti (1998) observaram baixa freqüência de K. schubartii no conteúdo alimentar de $C$. sapidus, atribuindo este fato à pouca profundidade em que vive este tanaidáceo, além da limitação de escavação apresentada por espécimes juvenis desse siri.

Apesar da contribuição de Decapoda ter sido menor do que os dois primeiros grupos, sua presença já era esperada e pode ser explicada pelo fato da existência de canibalismo e predação interespecífica, em condições ambientais, entre os braquiúros, principalmente sobre juvenis, indivíduos em ecdise ou doentes (ELNER, 1981; Williams, 1982; D'InCAO et al., 1990).

A constante presença do item Plantae no conteúdo alimentar do siri-azul caracteriza a espécie como generalista, já que também possui hábitos claramente carnívoros. DARnell (1958) observou C. sapidus comendo macrófitas vivas em Lake Pontchartrain, Lousiana, EUA. Para PAUL (1981), permanece a dúvida sobre a presença de vegetação nos estômagos de $C$. arcuatus e Callinectes toxotes Ordway, 1863, quanto à ingestão seletiva, acidental ou oriunda do trato digestivo das presas consumidas. Em exemplares adultos do caranguejo Necora puber, algas marrons ocorreram em $80 \%$ dos estômagos examinados, representando mais de $40 \%$ do volume total da dieta (CHOY, 1986).

BRANCO \& VERANI (1997) também reportaram material de origem vegetal no estômago de $C$. danae, porém esta espécie foi considerada como exclusivamente carnívora, já que a presença do material vegetal foi rara. Kapusta \& Bemvenuti (1998) também observaram filamentos e sementes de macrófitas em juvenis de $C$. sapidus. Plantas vasculares não-identificadas e algas também foram verificadas e freqüentemente estão no conteúdo estomacal de $C$. ornatus (Mantelatto \& Christofoletti, 2001).

Pode-se então concluir que material de origem vegetal faz parte da dieta de C. sapidus, comprovado por estudos realizados em juvenis desta espécie, que revelaram a capacidade fisiológica, uma vez que possuem as carbohidrases necessárias para metabolizar este material (McClinTOCK et al., 1991).

O item Annelida ocorreu em aproximadamente 36\% dos intestinos anteriores analisados (Tab. II), sendo um item alimentar de grande importância na dieta de Brachyura (BRANCO et al., 2002). Resultados similares foram obtidos por BRANCO \& VERANI (1997), sendo os poliquetos considerados por NonATO et al. (1990) um elo vital na cadeia trófica da região de Ubatuba, São Paulo. Este item também apresentou apenas restos e partes nãoidentificáveis em nível de espécie, contudo, sua freqüência de ocorrência foi representativa (Tab. II). KAPUSTA \& Bemvenuti (1998) observaram que duas espécies da infauna, os poliquetos Nephtys fluviatilis Monro, 1937 e Laeonereis acuta (Tredwell, 1923) foram freqüentes no conteúdo estomacal de juvenis de $C$. sapidus, enquanto que Heteromastus similis Southern, 1921, uma das espécies dominantes dos fundos moles e das enseadas estuarinas da Lagoa dos Patos (Bemvenuti, 1987), foi pouco freqüente. As freqüências de Annelida e Areia foram similares, demonstrando a relação existente entre estes dois itens. Esta relação pode representar a associação destes itens, já que os componentes de Annelida (poliquetos) têm como hábitat o sedimento.

Neste estudo, como em outros a respeito da dieta natural dos portunídeos, foi registrada a ocorrência de areia no conteúdo alimentar, porém este item não foi considerado como um componente alimentar. Esta presença pode ser atribuída à ingestão acidental (BRANCO \& VERANI, 1997), porém, uma parte desta ingestão seria feita como alimento devido à associação destas partículas com matéria orgânica (D'INCAO et al., 1990; CARQUEIJA \& GouvÊA, 1998). Outros autores já atribuem este fator a uma ingestão proposital, utilizada como fonte de minerais para a formação de uma nova carapaça (WILLLIAMS, 1982; CHOY, 1986). Este não é o caso dos siris analisados neste estudo, visto que todos estavam no estádio de intermuda. HAEFNER (1990) considerou areia como fonte de carbonato e o item mais freqüente na dieta de $C$. ornatus.

A ingestão pode estar também auxiliando em processos digestórios, através do contato mecânico, para a trituração de presas calcárias, como gastrópodes e bivalves (Dall \& Moriarty, 1983; Mantelatto \& Christofoletti, 2001). Isto pode ocorrer com os organismos analisados neste estudo, devido à alta freqüência do molusco bivalve $E$. mactroides.

A ingestão de areia por C. sapidus deve ocorrer juntamente com as presas, pois o siri-azul seleciona suas presas nos substratos, além de se enterrar. Além disto, a porcentagem de ocorrência do item Areia está muito próxima do item Anellida (Tab. II), reforçando a idéia de ingestão acidental. Assim, consideramos a ocorrência de areia no conteúdo alimentar do siri-azul como a atuação em conjunto da ingestão acidental e proposital.

$\mathrm{O}$ item Insecta apresentou baixo valor na freqüência de ocorrência em C. sapidus (Tab. II) e pode estar associada ao início do período de primavera (setembro/ outubro), quando estes organismos estão mais disponíveis no ambiente (D'INCAO et al., 1990; AsMus, 1998). A ingestão deve ocorrer acidentalmente, no momento em que o siri-azul está se alimentando de macrófitas aquáticas, ou mesmo quando estes organismos, já mortos, estão flutuando na superfície da água. Por outro lado, KAPUsta \& Bemvenuti (1998) não citaram a ocorrência deste item em juvenis de C. sapidus, enquanto BRANCO \& VERANI (1997) registraram em C. danae.

$\mathrm{O}$ item Pisces apresentou baixo valor na freqüência de ocorrência (Tab. II) em comparação com a freqüência de aproximadamente $88 \%$ para esta mesma espécie e $C$. similis, obtida por Hsuen et al. (1992) em Mobile Bay, Alabama. Os autores atribuem esta elevada freqüência a uma provável utilização, pelos siris, de restos de peixes descartados por redes de pesca. Este fato também foi relatado por PAUL (1981) para C. arcuatus, Branco (1996) para $C$. danae e CARqueiJa \& GouvêA (1998) para Callinectes larvatus Ordway, 1863.

$\mathrm{O}$ fato de não existir resto de peixes nos locais de coleta do siri-azul talvez explique a baixa freqüência observada neste estudo, até certo ponto similar às freqüências observadas na maioria dos estudos a respeito de aspectos da dieta natural de portunídeos. BRANCO \& 
VERANI (1997) observaram que, embora os peixes sejam um recurso abundante na Lagoa da Conceição e comumente consumido pelos siris, restam dúvidas de que C. danae possa capturar peixes de grande porte.

Por outro lado, West \& Williams (1986) sugerem que, em experimentos de inclusão de predadores, grandes espécimes do siri-azul (100-130 mm de comprimento de carapaça) podem capturar exemplares do peixe Fundulus similis (Baird \& Girard, 1853). Esta afirmação sustenta a idéia de que C. sapidus possa predar peixes na região estuarina da Lagoa dos Patos, uma vez que foram identificados, nos conteúdos alimentares de C. sapidus otólitos e restos ósseos, de peixes frequentemente encontrados no estuário da Lagoa dos Patos, como por exemplo, a corvina (Micropogonias furnieri (Desmarest, 1823)), que é, provavelmente, a espécie de maior biomassa do estuário da Lagoa dos Patos (VIEIRA et al., 1998).

A análise do conteúdo alimentar de $C$. sapidus nos diferentes meses de coleta não demonstrou mudanças significativas na dominância dos principais itens predados, indicando hábito alimentar definido (Fig. 2). Em março de 2003 a composição da dieta natural do siriazul foi bastante homogênea, não caracterizando nenhum item como o principal. O item Plantae merece destaque, por alcançar $80 \%$ na freqüência de ocorrência do conteúdo alimentar dos intestinos anteriores do siri-azul (Tab. III).

Nos meses seguintes, a dominância foi quase absoluta de E. mactroides, exceção a setembro de 2003, quando a dominância foi de Detrito (Tab. III). Esta baixa abundância deve-se ao intenso período de chuvas do ano anterior, tornando a região estuarina da Lagoa dos
Patos um ambiente dulcícola. $\mathrm{O}$ aumento dos valores de abundância deste bivalve ocorreu, provavelmente, devido ao fluxo de água doce vindo do norte da Lagoa em direção ao sul (Bemvenuti, 1998), trazendo juvenis de $E$. mactroides que posteriormente assentaram na região sul do estuário. Em maio de 2003, o destaque foi para o item Annelida (Tab. III); a alta porcentagem apresentada provavelmente está relacionada com a maior disponibilidade de organismos que têm nos meses de verão seu pico reprodutivo (BEMVENUTI, 1987), refletindo em valores de abundância elevados e indivíduos maiores nos meses seguintes.

Em julho de 2003, apesar do item Detrito não apresentar a maior porcentagem no método dos pontos, este item foi o que mais esteve presente no conteúdo alimentar de C. sapidus, ou seja, maior freqüência de ocorrência (Tab. III). Destaca-se o item Tanaidáceos, que apresentou maior porcentagem tanto do método dos pontos quanto na freqüência de ocorrência de todo o período estudado (Tab. III).

Setembro de 2003 foi caracterizado pela elevada porcentagem dos pontos para o item Insecta, a mais alta de todo o período de estudo (Tab. III). Vale ressaltar que foi a única ocasião em que este item obteve freqüência maior que Pisces. Ainda neste mês, Tanaidáceos apresentou valores elevados (menores do que aqueles observados em julho de 2003) nas porcentagens tanto dos pontos quanto das freqüências de ocorrência (Tab. III).

Em novembro de 2003 o item Outros Crustáceos apresentou valores elevados, porém menores do que aqueles observados em março de 2003 (Tab. III). Da mesma forma, o item Annelida também obteve valores elevados

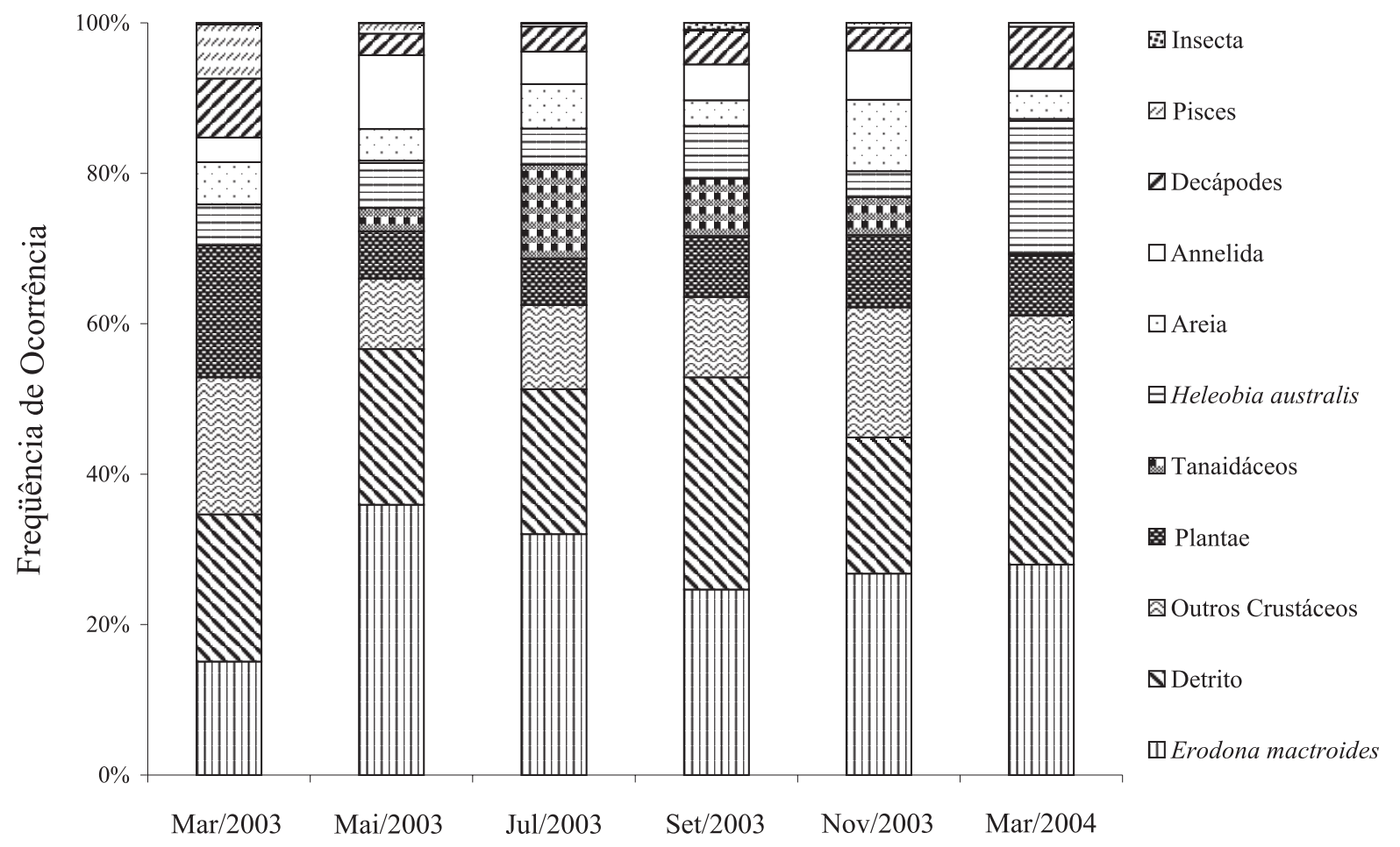

Fig. 2. Freqüência sazonal de ocorrência dos itens alimentares de Callinectes sapidus, durante o período de março de 2003 a março de 2004, na região estuarina da Lagoa dos Patos, Rio Grande, RS. 
Tabela III. Porcentagem sazonal dos Pontos (P\%) e Freqüência de Ocorrência (O\%) dos itens alimentares presentes nos intestinos anteriores do siri-azul Callinectes sapidus na região estuarina da Lagoa dos Patos, Rio Grande, RS.

\begin{tabular}{|c|c|c|c|c|c|c|c|c|c|c|c|c|}
\hline \multirow[t]{2}{*}{ Itens Alimentares } & \multicolumn{2}{|c|}{ Mar/2003 } & \multicolumn{2}{|c|}{$\mathrm{Mai} / 2003$} & \multicolumn{2}{|c|}{$\mathrm{Jul} / 2003$} & \multicolumn{2}{|c|}{ Set $/ 2003$} & \multicolumn{2}{|c|}{ Nov/2003 } & \multicolumn{2}{|c|}{ Mar/2004 } \\
\hline & $\mathrm{P} \%$ & $\mathrm{O} \%$ & $\mathrm{P} \%$ & $\mathrm{O} \%$ & $\mathrm{P} \%$ & $\mathrm{O} \%$ & P \% & $\mathrm{O} \%$ & $\mathrm{P} \%$ & $\mathrm{O} \%$ & $\mathrm{P} \%$ & $\mathrm{O} \%$ \\
\hline Erodona mactroides & 15,1 & 33,3 & 35,9 & 83,3 & 32,0 & 66,3 & 24,7 & 88,4 & 26,8 & 63,6 & 28,0 & 70,8 \\
\hline Detrito & 19,6 & 71,1 & 20,7 & 81,0 & 19,2 & 79,8 & 28,2 & 88,9 & 18,1 & 76,8 & 26,1 & 84,7 \\
\hline Outros Crustáceos & 18,2 & 66,7 & 9,4 & 48,4 & 11,2 & 35,8 & 10,7 & 40,1 & 17,3 & 55,2 & 7,1 & 25,0 \\
\hline Plantae & 17,6 & 80,0 & 6,3 & 26,2 & 6,2 & 28,5 & 8,1 & 34,3 & 9,6 & 46,8 & 8,1 & 43,1 \\
\hline Tanaidáceos & 0,0 & 0,0 & 3,1 & 16,7 & 12,5 & 36,3 & 7,6 & 26,6 & 5,0 & 18,4 & 0,1 & 1,4 \\
\hline Heleobia australis & 5,4 & 24,4 & 6,3 & 25,4 & 4,8 & 14,5 & 7,0 & 26,6 & 3,5 & 14,0 & 17,9 & 40,3 \\
\hline Areia & 5,6 & 44,4 & 4,2 & 30,2 & 5,9 & 42,5 & 3,4 & 17,9 & 9,5 & 38,4 & 3,7 & 23,6 \\
\hline Annelida & 3,3 & 15,6 & 9,8 & 48,4 & 4,3 & 32,6 & 4,8 & 25,6 & 6,5 & 44,8 & 3,0 & 29,2 \\
\hline Decápodes & 7,9 & 31,1 & 2,9 & 11,1 & 3,4 & 12,4 & 4,5 & 15,5 & 3,1 & 14,4 & 5,6 & 13,9 \\
\hline Pisces & 7,2 & 24,4 & 1,4 & 8,7 & 0,4 & 2,1 & 0,1 & 1,0 & 0,6 & 1,6 & 0,5 & 1,4 \\
\hline Insecta & 0,2 & 11,1 & 0,0 & 1,6 & 0,1 & 3,6 & 0,9 & 9,7 & 0,0 & 0,0 & 0,0 & 0,0 \\
\hline
\end{tabular}

(menores do que em maio de 2003), tanto nas porcentagens do método dos pontos, quanto na freqüência de ocorrência (Tab. III). Areia obteve a maior porcentagem do método dos pontos de todo o período estudado (Tab. III).

Por fim, vale ressaltar a maior porcentagem em março de 2004, tanto do método dos pontos, quanto na freqüência de ocorrência, observadas no item $H$. australis (Tab. III). Este item foi um dos quatro principais componentes da dieta natural de juvenis de $C$. sapidus, numa enseada da Lagoa dos Patos (KAPUSTA \& BEMVENUTI, 1998).

Sendo assim, Callinectes sapidus caracteriza-se como onívoro oportunista, com diversidade alimentar ampla e variações sazonais, porém não significativas $(\mathrm{p}>0,05)$, com tendência a predar invertebrados bentônicos, especialmente moluscos (bivalves e gastrópodes) e crustáceos (ostrácodes, tanaidáceos e restos de decápodes), assegurando, desta forma, sua manutenção na Lagoa dos Patos.

Agradecimentos. A Ana Carolina Tasch e Sandra Silvestre de Souza (bolsistas voluntárias, Fundação Universidade Federal do Rio Grande), pelo auxílio na triagem e identificação do material coletado. Ao Dr. Fernando Mantelatto, pela grande contribuição na revisão deste manuscrito.

\section{REFERÊNCIAS BIBLIOGRÁFICAS}

Asmus, M. L. 1998. A Planície Costeira e a Lagoa dos Patos. In: Seeliger, U.; Odebrecht, C. \& Castello, J. eds. Os ecossistemas costeiro e marinho do extremo sul do Brasil. Rio Grande, Ecoscientia. v.1, p.9-12.

Bemvenuti, C. E. 1987. Predation effects on a benthic community in estuarine soft-sediments. Atlântica 9(1):5-32.

1998. Invertebrados bentônicos. In: SeEliger, U.; Odebrecht, C. \& CAStello, J. eds. Os ecossistemas costeiro e marinho do extremo sul do Brasil. Rio Grande, Ecoscientia. v.1, p.46-51.

Blundon, J. A. \& Kennedy, V. S. 1982. Mechanical and behavioral aspects of blue crab, Callinectes sapidus (Rathbun), predation on Chesapeake Bay bivalves. Journal of Experimental Marine Biology and Ecology 65(1):47-65.

Boschi, E. E. 1982. Larvas de Crustacea Decapoda. In: Boltovskoy, D. ed. Atlas del zooplancton del Atlantico Sudocidental. Mar del Plata, INIDEP. p.699-758.

Branco, J. O. 1996. Variações sazonais e ontogênicas na dieta natural de Callinectes danae Smith, 1869 (Decapoda,
Portunidae) na Lagoa da Conceição, Florianópolis, S. C. Arquivos de Biologia e Tecnologia 39(4):999-1012.

Branco, J. O. \& Verani, J. R. 1997. Dinâmica da alimentação natural de Callinectes danae Smith (Decapoda, Portunidae) na Lagoa da Conceição, Florianópolis, Santa Catarina, Brasil. Revista Brasileira de Zoologia 14(4):1003-1018.

Branco, J. O.; Branco-Lunardon, M. J.; Verani, J. R.; SchVeitzer, R.; Souto, F. X. \& Vale, W. G. 2002. Natural diet of Callinectes ornatus Ordway, 1863 (Decapoda, Portunidae) in the Itapocoroy Inlet, Penha, SC, Brazil. Brazilian Archives of Biology and Technology 45(1):35-40.

Carqueija, C. R. \& GouvêA, E. P. 1998. Hábito alimentar de Callinectes larvatus Ordway (Crustacea, Decapoda, Portunidae) no manguezal de Jiribatuba, Baía de Todos os Santos, Bahia. Revista Brasileira de Zoologia 15(1):273-278

CEPERG/IBAMA. 2002. Desembarque de pescados no Rio Grande do Sul. Porto Alegre, Instituto Brasileiro do Meio Ambiente e dos Recursos Naturais Renováveis. sem paginação.

Chazaro-Olvera, S.; Rocha-Ramirez, A. \& Roman-Contreras, R. 2000. Observations on feeding, maturity, and fecundity of Callinectes similis Williams, 1966, on the central continental shelf off Veracruz, Gulf of Mexico. Crustaceana 73(3): 323-332.

Choy, S. C. 1986. Natural diet and feeding habits of the crabs Liocarcinus puber and L. holsatus (Decapoda, Brachyura, Portunidae). Marine Ecology Progress Series 31:87-99.

Dall, W. \& Moriarty, D. 1983. Functional aspects of nutrition and digestion. In: Mantel, L. H. ed. The biology of Crustacea. Internal anatomy and physiological regulation. New York, Academic. v.5, p.215-261.

DARNELL, R. 1958. Food habits of fishes and larger invertebrates of Lake Pontchartrain, Lousiana, an estuarine community. Publication of Institute Marine Science 5:353-416.

D’Incao, F.; Silva, K. G.; Rufino, M. L. \& Braga, A. C. 1990. Hábito alimentar do caranguejo Chasmagnathus granulata Dana, 1851 na Barra do Rio Grande, RS (Decapoda, Grapsidae). Atlântica 12(2):85-93.

Dittel, A. I. 1993. Cambios en los hábitos alimentarios de Callinectes arcuatus (Crustacea: Decapoda) en el Golfo de Nicoya, Costa Rica. Revista de Biologia Tropical 41:639-646.

EdGAR, G. J. 1990. Predator-prey interactions in seagrass beds. II. Distribution and diet of the blue manna crab Portunus pelagicus Linnaeus at Cliff Head, Western Australia. Journal of Experimental Marine Biology and Ecology 139(1-2):23-32.

Elner, R. 1981. Diet of green crab Carcinus maenas (L) from Port Hebert, southwestern Nova Scotia. Journal of Shellfish Research 1(1):89-94.

Fitz, H. C. \& Wiegert, R. G. 1990. Utilization of a Georgia salt marsh by the blue crab. Bulletin of Marine Science 46(1):244-251.

1991. Utilization of the intertidal zone of a salt marsh by the blue crab Callinectes sapidus: density, return frequency, and feeding habits. Marine Ecology Progress Series 
76(3):249-260

FreIRE, J. 1996. Feeding ecology of Liocarcinus depurator (Decapoda: Portunidae) in the Ría de Arousa (Galicia, northwest Spain): effects of habitat, season and life history. Marine Biology 126(2):297-311.

Freire, J. \& GonzÁlez-Gurriarán, E. 1995. Feeding ecology of the velvet swimming crab Necora puber in mussel raft areas of the Ría de Arousa (Galicia, NW Spain). Marine Ecology Progress Series 119:139-154.

HAEFner, P. A. 1990. Natural diet of Callinectes ornatus (Brachyura: Portunidae) in Bermuda. Journal of Crustacean Biology 10(2):236-246.

Hill, B. J. 1976. Natural food, foregut clearance-rate and activity of the crab Scylla serrata. Marine Biology 34(2):109-116.

Hines, A. H. \& Ruiz, G. M. 1995. Temporal variation in juvenile blue crab mortality: nearshore shallows and cannibalism in Chesapeake Bay. Bulletin of Marine Science 57(3):884-901.

Hines, A. H.; Haddon, A. M. \& Wiechert, L. A. 1990. Guild structure and foraging impact of blue crabs and epibenthic fish in a subestuary of Chesapeake Bay. Marine Ecology Progress Series 67(2): 105-126.

Hsueh, P.-W.; McClintock, J. B. \& Hopkins, T. S. 1992. Comparative study of the diets of the blue crabs Callinectes similis and $C$. sapidus from a mud-bottom habitat in Mobile Bay, Alabama. Journal of Crustacean Biology 12(4):615-619.

Hughes, R. N. \& SeEd, R. 1981. Size selection of mussels by the blue crab Callinectes sapidus: energy maximizer to time minimizer? Marine Ecology Progress Series 6(1):83-89.

Kapusta, S. C. \& Bemvenuti, C. E. 1998. Atividade nictemeral de alimentação de juvenis de Callinectes sapidus Rathbun, 1895 (Decapoda: Portunidae) numa pradaria de Ruppia maritima L. e num plano não vegetado, numa enseada estuarina da Lagoa dos Patos, R. S., Brasil. Nauplius 6:41-52.

Laughlin, R. A. 1982. Feeding habitats of the blue crab, Callinectes sapidus Rathbun, in the Apalachicola estuary, Florida. Bulletin of Marine Science 32:807-822.

Ludwig, J. A. \& Reynolds, J. F. 1988. Statistical ecology: a primer on methods and computing. New York, John Wiley \& Sons. 338p.

Mantelatto, F. L. M. \& Christofoletti, R. A. 2001. Natural feeding activity of the crab Callinectes ornatus (Portunidae) in Ubatuba Bay (São Paulo, Brazil): influence of season, sex, size and molt stage. Marine Biology 138(3):585-594.

McClintock, J. B.; Klinger, T. S.; Marion, K. \& Hsueh, P. 1991. Digestive carbohydrases of the blue crab Callinectes sapidus (Rathbun): implications in utilization of plant-derived detritus as a trophic resource. Journal of Experimental Marine Biology and Ecology 148(2):233-239.

McLaughlin, P. A. 1983. Internal anatomy. In: Mantel, L. H. ed. The biology of Crustacea. Internal anatomy and physiological regulation. New York, Academic. v.5, p.1-52.

McLaughlin, P. A. \& Hebard, J. F. 1961. Stomach contents of the Bering Sea King crab. Bulletin of the United States Fish Commission 5:5-8.

Melo, G. A. S. 1996. Manual de identificação dos Brachyura (caranguejos e siris) do litoral brasileiro. São Paulo, Plêiade/FAPESP. 604p.

Nonato, E. F.; Petti, M. A. \& Paiva, P. C. 1990. Contribuição dos anelídeos poliquetas na dieta de crustáceos decápodos braquiúros na região de Ubatuba. In: SiMPÓsIo DE ECOSSISTEMAS DA Costa Sul e Sudeste Brasileira, 2º Águas de Lindóia, 1990. Anais... São Paulo, ACIESP. p. 224-234.
Paul, R. G. K. 1981. Natural diet, feeding and predatory activity of the crabs Callinectes arcuatus and $C$. toxotes (Decapoda, Brachyura, Portunidae). Marine Ecology Progress Series 6:91-99.

Reise, K. 1985. Tidal Flat Ecology. Berlin, Springer-Verlag. $191 \mathrm{p}$.

Rosa-Filho, J. S. \& Bemvenuti, C. E. 1998. Caracterización de las comunidades macrobentónicas de fondos blandos en regiones estuarinas de Rio Grande do Sul (Brasil). Thalassas 14:43-56.

Rosas, C.; Lazaro-Chaves, E. \& Bückle-Ramirez, F. 1994. Feeding habitats and food niche segregation of Callinectes sapidus, $C$. rathbunae and $C$. similis in a subtropical coastal lagoon of the Gulf of Mexico. Journal of Crustacean Biology 14(2):371382 .

SchaffNer, L. \& Diaz, R. J. 1988. Distribution and abundance of overwintering blue crabs, Callinectes sapidus, in the Lower Chesapeake Bay. Estuaries 11(1):68-72.

SEED, R. 1980. Predator-prey relationships between the mud crab Panopeus herbstii, the blue crab Callinectes sapidus and the Atlantic ribbed mussel Geukensia (=Modiolus) demissa. Estuarine Coastal and Shelf Science 11:445-458.

Sih, A.; Crowley, P.; McPeek, M.; Petranka, J. \& Strohmeier, K. 1985. Predation, competition, and prey communities - a review of field experiments. Annual Review of Ecology and Systematics 16:269-311.

Stoner, A. W. \& Buchanan, B. A. 1990. Ontogeny and overlap in the diets of four tropical Callinectes species. Bulletin of Marine Science 46(1):3-12.

Tagatz, M. E. 1968. Growth of juvenile blue crabs, Callinectes sapidus Rathbun, in the St. Johns River, Florida. Fishery Bulletin 67(2):281-288.

Taissoun, E. N. 1969. Las especies de cangrejos del genero "Callinectes" (Brachyura) en el Golfo de Venezuela y Lago Maracaibo. Boletin del Centro de Investigaciones Biologicas 2:1-112.

Vieira, J. P.; Castello, J. P. \& Pereira, L. E. 1998. Ictiofauna. In: Seeliger, U.; Odebrecht, C. \& Castello, J. eds. Os ecossistemas costeiro e marinho do extremo sul do Brasil. Rio Grande, Ecoscientia. v.1, p.60-68.

Wear, R. G. \& Haddon, M. 1987. Natural diet of the crab Ovalipes catharus (Crustacea: Portunidae) around central and northern New Zealand. Marine Ecology Progress Series 35:39-49.

West, D. \& Williams, H. 1986. Predation by Callinectes sapidus (Rathbun) within Spartina alterniflora (Loisel) marshes. Journal of Experimental Marine Biology and Ecology $100(1-3): 75-95$

Williams, A. B. 1974. The swimming crabs of the genus Callinectes (Decapoda: Portunidae). Fishery Bulletin 72(3):685-798.

Williams, M. J. 1981. Methods for analysis of natural diet in portunid crabs (Crustacea: Decapoda: Portunidae). Journal of Experimental Marine Biology and Ecology 52(1):103-113. . 1982. Natural food and feeding in the commercial sand crab Portunus pelagicus Linnaeus, 1766 (Crustacea: Decapoda: Portunidae) in Moreton Bay, Queensland. Journal of Experimental Marine Biology and Ecology 59(2-3):165-176.

Wright, R. A.; Crowder, L. B. \& Martin, T. H. 1996. Selective predation by blue crabs on the gastropod, Bittium varium: confirmation from opercula found in the sediments. Estuaries 19(1):75-81

ZAR, J. H. 1984. Biostatistical Analysis. 2 ed. New Jersey, Prentice Hall. 718p. 